\title{
Review of Research Studies on Biomechanics of Swallowing and Dysphagia
}

\author{
Joana Almeida ${ }^{1}$, Renato Natal Jorge ${ }^{2}$ \\ ${ }^{1}$ Doctoral Program in Biomedical Engineering, Faculty of Engineering, University of Porto, \\ Porto, Portugal (jlopesalm@gmail.com) ORCID 0000-0003-4248-0586; ²LAETA, INEGI, Faculty \\ of Engineering, University of Porto, Porto, Portugal (rnatal@fe.up.pt) ORCID 0000-0002-7281- \\ $579 x$
}

\begin{abstract}
Dysphagia is a prevalent condition characterised by the dysfunction/ incoordination of the anatomical structures involved in the swallowing biomechanics.

Dysphagia can have serious clinical consequences such as pneumonia, malnutrition, dehydration and even death. It implies a high negative impact on patients quality of life and may also represent increased financial costs in order to treat associated complications.

It is essential to correctly identify and evaluate patients with this problem. However, the gold standard diagnostic exams present risk of aspiration, they are uncomfortable and invasive, and still present other inherent disadvantages/risks related with the technique itself.

The simulation area may contribute to a more detailed knowledge of the swallowing biomechanics and, when applied to pathologic scenarios (i.e. dysphagia), to identify higher risk patients and manage the treatment in a more adequate and specific way. It will also seems to be able to reduce the use of invasive techniques, excessive radiation and associated economical costs.

The aim of this paper is to review the existing research studies on biomechanical mechanisms of swallowing and dysphagia.

Computer simulation seems to be a solution to better understand these mechanisms and even to reduce clinical disadvantages when compared with the available diagnostic methods used with these patients.
\end{abstract}

Author Keywords: Swallowing, Dysphagia, Swallowing Simulation, Numerical Simulation, Three-Dimensional Organ Modelling.

Type: Review Article

əे Open Access $\square$ Peer Reviewed @C) CC BY

\section{Introduction}

Dysphagia is described as an abnormality and symptom characterised by a difficulty during the swallowing process. It is a prevalent condition, recognised by the World Health Organisation (WHO) in the International Classification of Disease (ICD) (Newman et al. 2016). Dysphagia can be present as a consequence of various pathologic conditions. There are examples such as older people (prevalence between 15-40\%), neurodegenerative diseases (Parkinson: 52-82\%; Alzheimer: 57-84\%; motor neuron disease, depending on the stage of the disease: $30-100 \%)$, stroke (37-78\%), traumatic brain injury (25\%), neck and head cancer as disease consequence or after chemotherapy (44-50\%) (Hayoun et al. 2015; Mowlavi et al. 2016; Newman et al. 2016).

\subsection{Anatomy \& physiology of swallowing process}

The oral cavity is separated from the pharynx by the faucial pillars. The pharynx, in its turn, is delimited by pharyngeal constricting muscles that are inserted superiorly in the skull and hyoid 
bone, anteriorly in the thyroid cartilage and posteriorly in the median raphe. The submental muscles are originated in the mandible and inserted in the hyoid bone and in the tongue. The cricopharyngeus muscle, which is inserted anteriorly in the cricoid cartilage, encloses the upper oesophageal sphincter (UES). The epiglottis, located in the larynx, is inserted anteriorly into the hyoid and thyroid cartilage and is angled superior and posteriorly. The space between the surface of the tongue and the epiglottis is designated as vallecula. In the laryngeal vestibule are located the true and false vocal cords, as well as the laryngeal surface of the epiglottis. Two spaces designated as piriform recesses are located lateral to the larynx (Matsuo and Palmer 2008). The main anatomical structures are represented in Figure 1.

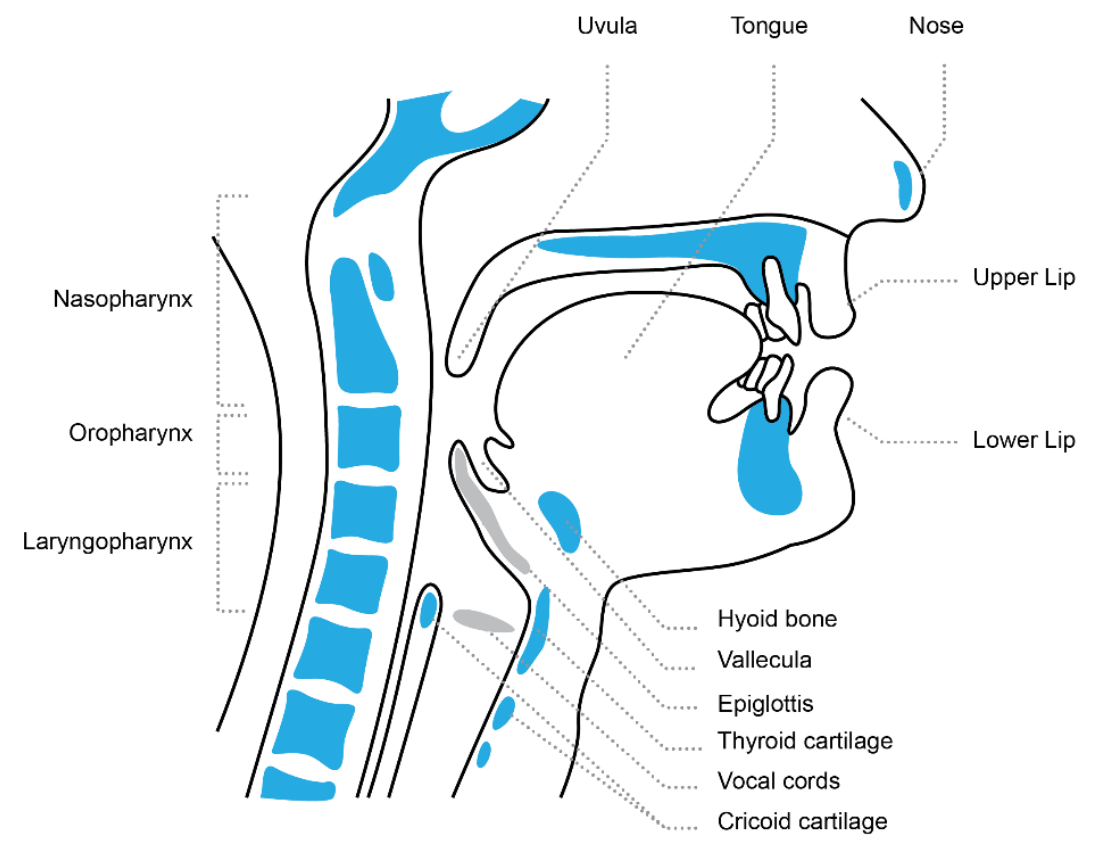

Figure 1: Simplified representation of the anatomical structures involved in swallowing process

The process of liquid swallowing is generally divided into four phases, according to the anatomical location of the bolus and the underlying biomechanics: oral preparatory, oral propulsive, pharyngeal and oesophageal stage (Matsuo and Palmer 2008).

During the oral preparatory phase, food is conducted into the oral cavity and manipulated (chewed and mixed with saliva) in order to obtain a food bolus ready to be swallowed. During this phase, the velopharyngeal sphincter is open but the posterior oral cavity is closed by tongue-palate contact (Nicosia 2007; Matsuo and Palmer 2008; Fairfield et al. 2010).

The oral propulsive phase occurs with the posterior ejection (i.e. propelled by the tongue) of the bolus to the pharynx. The velopharyngeal sphincter is closed during this phase, preventing nasal food regurgitation (Nicosia 2007; Fairfield et al. 2010).

The reflex pharyngeal phase begins when the food bolus contacts the base of the tongue. The hyoid bone and the larynx move both upward and anteriorly, leading to a pressure differential that drives the bolus in the pharynx direction. These structures movement (hyoid and larynx) contribute to trachea and vocal cords closure and also to the retroflexion epiglottis movement (Nicosia 2007; Fairfield et al. 2010). This phase is described in more detail in the next subsection (vid. subsection 1.2.).

The oesophageal phase is also a reflex phase, during which the bolus is conducted through the inferior oesophageal sphincter by a peristalsis movement (Fairfield et al. 2010). 
The swallowing of solids is more correctly described by the Process Model of Feeding and includes an additional stage, counting on a total of five stages: transport I, food processing, transport II, pharyngeal and oesophageal stage (Matsuo and Palmer 2008). Only the first three stages significantly differ from the above described liquid stages.

Transport I stage begins with the food conduction to the oral cavity. The post-canine region drives the food into the occlusal teeth region (Matsuo and Palmer 2008).

Food processing stage consists in food mastication and saliva mixture until an optimal consistency is reached in order to be swallowed. Differently from the liquids corresponding stage (i.e. "oral preparary stage"), the posterior oral cavity is open (i.e. in communication with pharynx) which allows the aroma contact with nose chemoreceptors (Matsuo and Palmer 2008).

Transport II stage (corresponding to liquids "oral propulsive stage") can occur intermittently with food processing cycles (Matsuo and Palmer 2008).

\subsection{Swallowing or breathing - a vital coordination during the pharyngeal stage of swallowing}

During pharyngeal stage two biologically meaningful events must occur in a highly synchronized sequence: the flow of the food bolus towards the pharynx and the airway protection (while the first event occurs). Consequently, it is a crucial phase for human swallowing during which, in pathological scenarios (i.e dysphagia), pulmonary aspiration can be present (Matsuo and Palmer 2008).

During the pharyngeal stage, and while the bolus is driven to the pharynx, the soft palate encloses the nasopharynx through a lifting movement and its contact with the posterior and lateral walls of the pharynx (Matsuo and Palmer 2008). This event is represented in Figure 2.

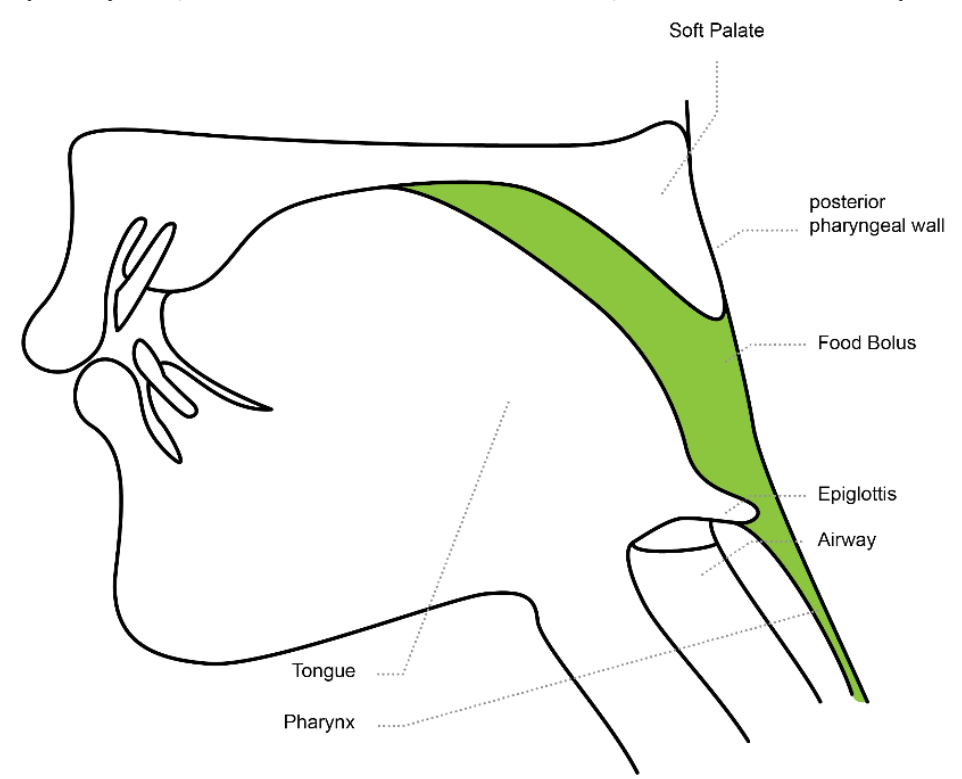

Figure 2: Pharyngeal stage during which a bolus (green) is driven towards the pharynx

The retraction movement of the base of the tongue and the sequential contraction of the constricting muscles of the pharynx shorten the pharynx and contribute to the propulsion of the bolus into the oesophagus. This stage lasts 1 second, during which two important events occur simultaneously: the passage of the food bolus (driven from the pharynx to the oesophagus) and the protection of the airway (isolation of the larynx and trachea during the passage of this bolus). Thus, during this stage, a short apnea occurs (interruption of pulmonary 
ventilation for 0.5 - 1.5 seconds), which is a consequence of the closure of airway, resulting from elevation of the soft palate and the tilt movement of the epiglottis, as well as neural suppression occurring in the brainstem (Matsuo and Palmer 2008).

After the swallowing of a liquid bolus, the breath resumes with an expiration movement. This mechanism prevents aspiration. However, during sequential swallowing, breathing can resume with an inspiration movement. During solid food swallowing, the breathing pauses are longer and begins significantly earlier than the onset of swallowing (Matsuo and Palmer 2008).

\subsection{Airway protective mechanisms during swallowing}

There are several mechanisms that ensure a safe transition of the food bolus to the pharynx, and then to the oesophagus, without aspiration (without bolus airway entry). These mechanisms include vocal cord closure, posterior tilt movement of the arytenoids, anterior and superior excursion of the hyoid and the larynx (through contraction of the suprahyoid and thyroid muscles), and the tilt movement of the epiglottis (Matsuo and Palmer 2008).

The posterior tilt movement of the epiglottis contributes to the isolation/closure of the laryngeal vestibule. This mechanism is described by several authors, although their opinion regarding a more detailed kinematic analysis is not yet consensual (Fink, Martin, and Rohrmann 1979; Ekberg and Sigurjónsson 1982; Vandaele, Perlman, and Cassell 1995; Garon et al. 2002; Matsuo and Palmer 2008; Seo et al. 2014; Pearson et al. 2016).

Mizunuma et al. (2009) highlighted that any numerical simulation of swallowing must take into account epiglottis retroflexion movement and larynx elevation because these structure movements have a crucial role in a safe swallowing.

Seo, Oh, and Han (2016) did a quantitative analysis of swallowing pattern movements and explored kinematic factors related to penetration-aspiration. These authors concluded that there is an association between risk of aspiration and alteration of movement of the epiglottis.

Regarding the value of the epiglottis mechanism for the swallowing process, Perlman, Booth, and Grayhack (1994) concluded that patients with alterations in this mechanism present a four times higher risk of aspiration when compared to patients without alterations. These authors found that $56 \%$ of the patients with alterations in the epiglottis mechanism aspirate, whereas only $18 \%$ of patients without alterations present aspiration.

Garon et al. (2002) also highlighted the importance of epiglottic biomechanics analysis as one of the parameters to be considered during a videofluoroscopic swallowing exam. These authors found a $59 \%$ aspiration rate and a $31 \%$ penetration rate in patients with alterations of the epiglottis mechanism.

\subsection{Clinical implications of dysphagia}

About $50-75 \%$ of patients with dysphagia present bolus penetration of laryngeal vestibule, which means the entrance of food material in the laryngeal vestibule, above the vocal cords level. Between $20-25 \%$ of these penetrations result in airway aspirations (passage of food material below the vocal cords) (Newman et al. 2016).

The presence of food material in the lung area can induce respiratory complications including aspiration pneumonia. Dysphagia is considered the major cause of pneumonia after stroke, affecting about $20 \%$ of these patients, and causing more than $10 \%$ of deaths after stroke. Dysphagia can also result in other clinical complications, such as malnutrition and dehydration up to $25 \%$ of patients after stroke (Hayoun et al. 2015; Mowlavi et al. 2016; Newman et al. 2016). 
The fast identification of a patient with dysphagia, and its detailed evaluation is, therefore, important to avoid these serious clinical consequences. The more precise knowledge of the altered mechanisms involved in the swallowing process may also allow drive the rehabilitation treatment of these patients in a more specific way (e.g.: identifying and targeting rehabilitation to a specific group of muscles) (Matsuo and Palmer 2008).

\subsection{Current forms of diagnostic and evaluation of dysphagia}

Dysphagia can be assessed by clinical judgment, requiring anamnesis data, and can include the use of standardised scales. Some of these scales include the swallowing of bolus of different volumes and viscosities. An additional data, in particular about the risk of aspiration, can still be provided with the use of a pulse-oximeter (Vilardell et al. 2017).

Some of the clinical signs of an impaired swallow consist in the presence of oral residue (part of the bolus remains in the oral cavity after the swallow), impaired labial seal, symptoms of pharyngeal residue (e.g. cough, sensation of a pharyngeal foreign body presence), fractional swallows (multiples swallows for the same bolus), changes in voice quality (e.g. wet voice) and decrease in oxygen saturation $\geq 3 \%$ than the basal level (Vilardell et al. 2017). This clinical evaluation depends on the clinician's knowledge (and experience) in anatomical structures and neurophysiologic processes involved in swallowing. Thus, it is a very subjective evaluation and has several inter-individual differences (Spadotto et al. 2008).

There are two popular instrumental techniques for dysphagia diagnosis and evaluation, considered as gold standard: videofluoroscopic swallowing study (VFSS), also known as the Modified Barium Swallow (MBS), and fiberoptic endoscopic evaluation of swallowing (FEES) (Langmore 2017; Vilardell et al. 2017). The first one (VFSS) is a dynamic radiologic video technique that measures, in real-time, spatial-temporal abnormalities in the biomechanics of the swallowing process. The images are two-dimensional and are defined by the interaction between X-rays, barium contrast added to a swallowed bolus and the different densities of the oropharyngeal anatomical structures. There are softwares (e.g.: Swallowtail ${ }^{\circledR}$ ), described in the literature, for acquisition of quantitative parameters, which allow a more objective analysis of the swallowing process (Belldev Medical, n.d.). However, there is an inevitable radiation exposure that limits the repetition of this exam, as well as studies in healthy subjects. The risk of contrast aspiration is also real (Spadotto et al. 2008; Mizunuma et al. 2009; Ono et al. 2009; Mowlavi et al. 2016; Newman et al. 2016). Furthermore, it is unknown the predictive power of this technique to a complete meal (Adnerhill, Ekberg, and Groher 1989). This exam has a limited resolution of the soft-tissues as the tongue (Chang, Rosendall, and Finlayson 1998; Tasko, Kent, and Westbury 2002; Newman et al. 2016). It also provides insufficient data regarding the applied forces on the bolus (Chang, Rosendall, and Finlayson 1998). Authors believe that swallowing process anomalies are commonly under diagnosed with this technique (Nicosia and Robbins 2001).

Barium mixtures normally used in the VFSS are three times denser than liquids without barium, such as those normally ingested. This difference leads to a difficult extrapolation of the results obtained with this exam. It becomes clear that the clinicians need a better understanding of the properties manipulation effect of the swallowed materials (Dantas et al. 1989; Nicosia and Robbins 2001).

The second technique (FEES) consists in the insertion of an optical fibre cable, through the nose, that allows the direct visualisation of a previous coloured swallowed bolus. During FEES, some authors state that the cable insertion can interfere with the normal bolus flow and, consequently, with the normal swallow physiology (Ono et al. 2009; Mowlavi et al. 2016; 
Newman et al. 2016). During the second half of this examination there is a "white-out" phase due to the oropharyngeal structures collapse, in which the visualisation is momentarily interrupted. Other authors still point some possible clinical complications as epistaxis, vasovagal response, and laryngospasm. However, these complications are very uncommon and spontaneously resolved, and FEES is considered an extremely secure technique (Langmore 2017).

However, both techniques (VFSS and FEES) are uncomfortable, invasive and include the risk of food/liquid aspiration (Ono et al. 2009; Mowlavi et al. 2016; Newman et al. 2016; Langmore 2017).

Manometry, other possible technique described in the literature, allows the pressure measurement during the pharyngeal bolus transportation, with an adequate spacial and temporal resolution. However, this pressure measurement can be incorrect due to the difference between the hydrodynamic pressure, during the passage of bolus through the transducer, and the contact pressure, when the pharyngeal wall contracts above the transducer. It is the hydrodynamic or the intra-bolus pressure that controls the bolus transport. Moreover, it is believed that the catheter presence causes discomfort (in the nose and oropharynx) and interrupts the normal bolus flow. The elevation movement of the larynx and the shortening of the pharyngeal wall can induce the catheter displacement and, consequently, raise doubts about the precise local where the pressures are being measured (Chang, Rosendall, and Finlayson 1998).

In the literature it is possible to find other described methods, less commonly used in clinical practice, such as ultrasounds, acoustic analysis of swallow, tissue doppler imaging and surface electromyography.

The use of ultrasounds in the evaluation of dysphagia is a non-invasive fast method with low cost involved and it is possible to be realised at bedside. However, it does not exclude the need of other methods since it cannot identify some dynamic components, as for example, food stasis inside the pharynx. As so, it is not a very used technique (Leite et al. 2014).

There are some scientific papers about the acoustic analysis method of swallow sounds. This technique is performed with the use of a microphone and a notebook computer system. However, the authors highlight that data obtained from this analysis should be included in the clinical bedside evaluation and should not replace other diagnostic methods (Santamato et al. 2009).

A new dysphagia screening method is described in the literature - tissue doppler imaging. Authors present this method as a way of evaluating abnormalities of the esophageal wall movements (Manabe et al. 2018).

There is also an evaluation method that uses surface electromyography. It is a non-invasive and inexpensive method that measure the activity of some muscular groups involved in the swallowing process, as masseter, submental, and infrahyoid groups (Poorjavad et al. 2017). However, it does not allow us to have an image view of the swallowing process.

\subsection{Thickeners and other treatment strategies for dysphagia}

One of the most common forms of dysphagia treatment is the use of thickeners, in order to increase viscosity of liquid drinks (shear-thinning liquids) and, consequently, the safety of the patients during food and medication ingestion as well as of their hydration (Nicosia 2007; Mowlavi et al. 2016; Newman et al. 2016).

Thickeners are used to modify fluids properties and, traditionally, they are composed by modified amide granules that are carbohydrates with capacity to absorb water and swell, 
resulting in a higher viscosity liquid. However, they have some limitations as the amide taste, often reported as unpleasant, and a grainy texture (Newman et al. 2016).

Nowadays, there are some new thickeners molecules (xanthan gum) which, in comparison with the old amide thickeners, have better palatability. They are not digested by amilase, which allows them to maintain the same viscosity until completely swallowed, and they are potentially able to ensure a better hydration once they reduce the extracted water needed to thicken liquids (Newman et al. 2016).

Other usual forms of dysphagia treatment are the volume control of the swallowed food/liquids and the modification of the applied forces to the bolus, through cervical postural or pharyngeal cleaning manoeuvres, in an attempt to contribute to a safer swallowing (reducing the aspiration risk) (Chang, Rosendall, and Finlayson 1998).

\section{Summary of biomedical simulation on dysphagia}

Computational models may offer several advantages for the analysis of the swallowing process once they allow to predict the result without exposing patients to uncomfortable techniques or excessive radiation, as in the case of the most common complementary diagnostic tests in dysphagia (i.e. FEES and VFSS). In addition to the mentioned clinical advantages, computer simulation could drastically reduce institutional costs associated with these complementary diagnostic tests. By reducing the impact of these disadvantages (clinical and economical), this analysis may become accessible to all patients with dysphagia as well as doable by all institutions whereas specified technical professional would not be required.

Over the years, several studies have been developed in this area, as summarised in the timeline (Figure 3).

In 1993, Kahrilas, Lin, Logemann, Ergun and Facchini matched biplane videofluoroscopic images and manometry to evaluate the propulsion of different bolus volumes and the tongue action (Kahrilas et al. 1993).

In 1996, Kahrilas, Lin, Chen and Logemann presented a tridimensional reconstruction of accommodation mechanism during pharyngeal swallowing through four valves. These valves open/close as the pharynx works as an airway or a swallowing channel: glossopharyngeal junction (GPJ), velopharyngeal junction, laryngeal vestibule and UES (Kahrilas et al. 1996).

Chang, Rosendall, and Finlayson (1998), presented a mathematical model of the bolus movement through the pharynx. They used data (pharyngeal walls and UES movements) from videofluoroscopic swallowing images. These authors described the sectional shape of the pharynx as being ellipsoidal (Chang, Rosendall, and Finlayson 1998). In the following year, Chang, Lin, and Hwang (1999) developed an algorithm, which they called K-SNAKE, as a formula to solve the active contours of the deformable models.

Nicosia and Robbins (2001) presented a mathematical model based on parallel plate squeezing. They analysed the effect of viscosity and tongue action on the dynamics of oropharyngeal bolus ejection. These authors also included, for the first time, the analysis of density effect. 


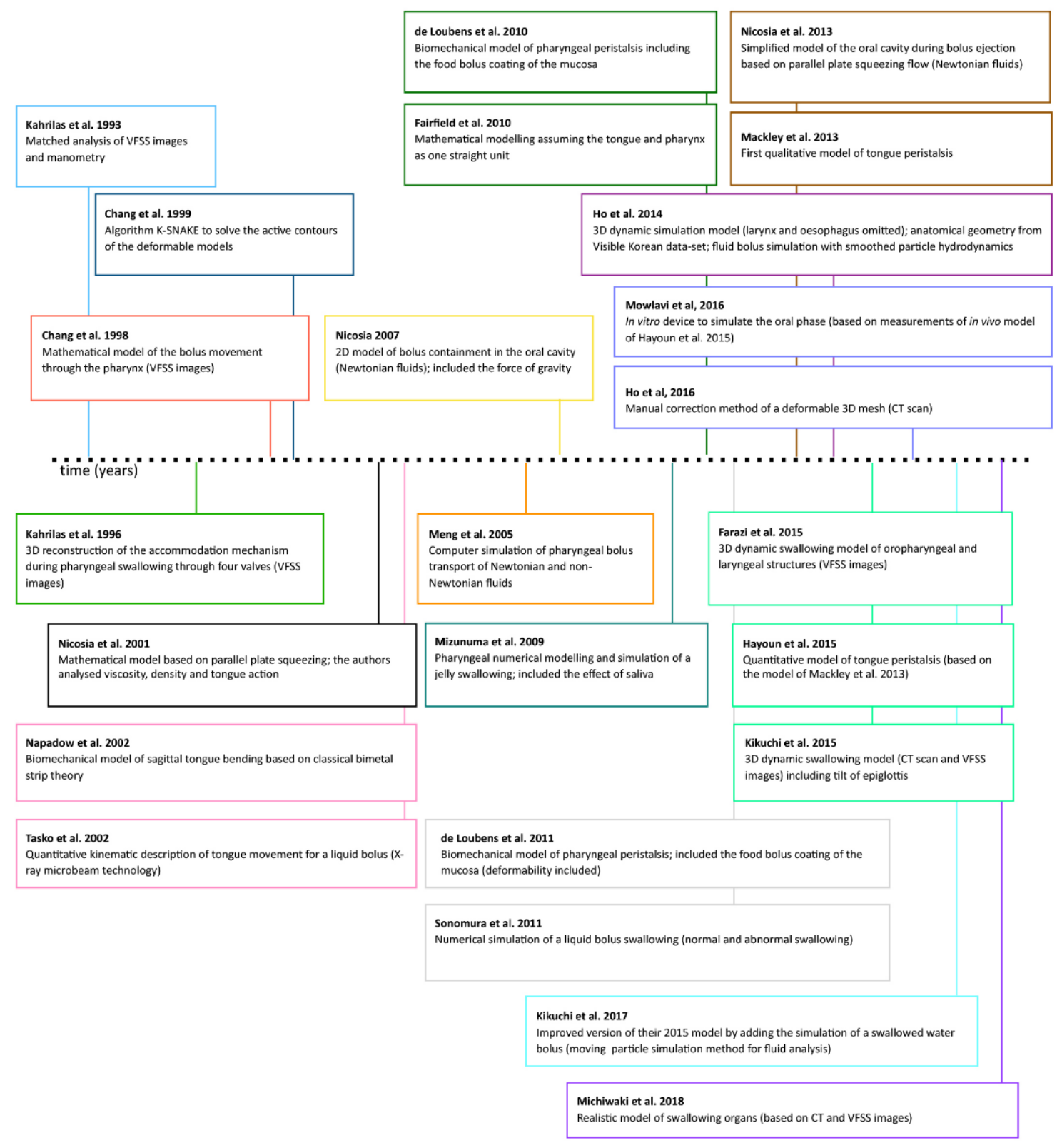

Figure 3: Timeline of the most relevant studies on dysphagia simulation

Napadow, Kamm, and Gilbert (2002) studied the biomechanics of the tongue, using the simulation of muscle contraction based on the bimetal strip model. The characteristics of the model were extrapolated from magnetic resonance imaging (MRI) and from Visible Human: Male database.

Tasko, Kent, and Westbury (2002) presented a kinetic description of the lingual movements. They used small gold pellets attached to the tongue, mandible and other areas of the oral cavity which were then tracked by an $\mathrm{x}$-ray microbeam technology while subjects swallowed water bolus.

Meng, Rao, and Datta (2005), simulated the movements of the pharyngeal walls and analysed the rheological properties of Newtonian and non-Newtonian shear-thinning fluid bolus. To define boundaries, they used data published by Kahrilas et al. (1993) and Cook et al. (1989). Nicosia (2007) presented a two dimensional computational model to analyse the bolus containment of Newtonian fluids in the oral cavity, defending the idea that this is a mechanism highly influenced by the thickening of fluids. The author presented a hybrid computational 
strategy designated by Arbitrary Lagrangian-Eulerian (ALE), due to being able to handle wide grid distortions and used the fluid "sloshing" in a partially filled tank, which is oscillating from side-to-side as a validation method. The geometric model was based on data already published by Kahrilas et al. (1993).

Mizunuma et al. (2009) presented a numerical swallowing model of a jelly limited to the pharyngeal phase. They emphasised the importance of including epiglottis retroflexion movement and larynx elevation because these are events with a crucial role for a safe swallowing. They also mentioned that the model should include solid and liquid food since they have different mechanical characteristics. They pointed out that saliva plays an important role in reducing the frictional properties of the viscous bolus and thus included this effect in the model (Mizunuma et al. 2009).

Fairfield et al. (2010) presented a mathematical modelling assuming the tongue and pharynx as one straight unit. They considered three different bolus types: liquid, partial liquid/solid and solid. They estimated that gravity was not relevant to the transition during the first three stages (oral preparatory, oral, and pharyngeal stage) while inertia and viscosity were essential for bolus propulsion.

De Loubens et al. (2010) developed a biomechanic model of pharyngeal peristalsis that included the food bolus coating of the mucosa, so that they could analyse the impact of food bolus viscosity on flavour release. These authors assumed that a thin film of saliva lubricates the mucosa. They also considered the hypothesis of a constant hydrodynamic regime.

In 2011 the same authors presented some improvements to the previous model, considering deformability. They developed an elastohydrodynamic model of pharyngeal peristalsis and compared predictions with in vivo observations for Newtonian fluids (de Loubens et al. 2011). Sonomura et al. (2011) presented a finite element swallowing model (normal and abnormal swallowing) of a liquid bolus. They analysed the viscous properties and volume of the bolus. These authors simulated two types of changes during the swallowing process: a) the dysfunction in the lifting up of the throat and retroflexion of the epiglottis (important movements to prevent aspiration); b) the early termination of these movements. Aspiration risk was present in both cases, but the bolus flow was different. This model included some improvements to the 2009 model (Mizunuma et al. 2009): the solid elements were replaced by shell elements, the tongue was composed of two parts and it was included a soft palate. These authors observed that the swallowing flow of the liquid bolus was significantly influenced by the following factors: gravitational force on the bolus, peristalsis-like movement of the pharynx walls, the flow resistance and the frictional resistance between the bolus and the pharynx. Since the frictional resistance was not modulated in this simulation, the authors chose to reduce the gravitational force. By that, they corrected the excessive bolus velocity and the repulsive force was calculated to inhibit the penetration of the bolus material (Sonomura et al. 2011).

Nicosia (2013) used a model of the bolus ejection from the oral cavity, based again, according to this author, on the parallel plate squeezing flow, in order to quantify the shear rate of Newtonian boluses of different viscosities. He assumed that the results were also extensible to non-Newtonian fluids and that saliva would experience a no-slip condition at the tongue interface.

In 2013, Mackley, Tock, Anthony, Butler and Chapman characterised the rheology of liquid thickeners and presented the first qualitative model of tongue peristalsis (Mackley et al. 2013). 
In 2014, Ho, Tsou, Green and Fels constructed a three-dimensional computer simulation of swallowing using finite element models to simulate soft structures in which they omitted larynx and oesophagus. Bony structures were simulated as rigid bodies. Geometry was based on Visible Korean data-set. The trajectory movements of these organs were not based on medical images. Fluid bolus of two different viscosities were simulated with smoothed particle hydrodynamics (SPH). These authors did not included the gravity effect (Ho et al. 2014).

The authors also observed that less viscous bolus is ejected from oral cavity with a higher velocity than "honey-like" bolus, consequently entering the nasopharynx. Some residues from "honey-like" bolus persisted in the oral cavity, oropharynx and nasopharynx. These authors also suggested that future works should include the gravity effects and the simulation of the lubricating saliva layer. They concluded that SPH seems to be a useful tool to simulate the bolus mass (Ho et al. 2014).

Kikuchi et al. (2015) obtained a motion model with forced displacement control regions. Softtissue organs were simulated as nonlinear elastic material with the Hamiltonian moving particle method (MPS). These authors analysed the behaviour of the tongue, palate, pharynx, oesophagus and larynx using CT scan (computational tomography scan) and VFSS images. They examined the retroflexion mechanism of the epiglottis, assuming this as an essential mechanism to prevent aspiration. For this last analysis they obtained four different motion models of the epiglottis and friction coefficients (Kikuchi et al. 2015).

The results obtained by Kikuchi et al. (2015) suggested that there is a strong correlation between a dysfunctional epiglottis movement and risk of aspiration.

In 2015, Farazi, Martin-Harris, Harandi, Fels and Abugharbieh presented a three dimensional biomechanics swallowing model of the oral, pharyngeal and laryngeal muscles and structures. They used geometric and kinematic data from the swallowing animations of the Modified Barium Swallow Impairment Profile ${ }^{\circledR}$ protocol. These animations were created from real videofluoroscopic images. These authors simulated a mixture of rigid bodies (jaw and teeth) and soft structures (tongue and soft palate). They used a smoothed particle hydrodynamics simulation and considered two different viscosities: thinner and thicker liquid. They established an initial density and no-slip condition. Gravitational force was also included. The average human tongue measurements reported by Hopkin (1967) was used to scale this model (Farazi et al. 2015).

Hayoun et al. (2015) presented improvements to the model of Mackley et al. (2013) to simulate and quantify the oral stage of swallowing. They suggested that this model simulates in a more physiological way the swallowing process than the model of Nicosia and Robbins (2001). In their model, they included an adjustable gap between the tongue and the palate. This gap represents the tongue peristalsis movement (tongue-palate sealing) during the oral phase of swallowing.

Mowlavi et al. (2016) presented an in vitro swallowing simulator to measure and compare the swallowing of different types of liquids. They considered the flow of Newtonian and thickened fluids. These authors made some improvements to the model of Hayoun et al. (2015), adding the use of in vivo observations and the possibility of control the inertia resulting from all tissues and organs present in the swallowing process. They also considered the gravity effect, not previously included by Hayoun et al. (2015) (Mowlavi et al. 2016).

Ho et al. (2018) presented a manual method for the creation of a deformable 3D mesh based in human oropharyngeal swallow CT scan images. This dynamic mesh can be used with fluid 
simulation using SPH method. The simulation included the oral cavity, pharynx, larynx, trachea, oesophagus and nasopharynx (Ho et al. 2018).

In 2017, Kikuchi, Michiwaki, Koshizuka, Kamiya and Toyama presented an improved version of their 2015 model, by adding the simulation of a swallowed water bolus flow and studying the interaction between organs and the fluid bolus. Bolus flow analysis was performed with an explicit MPS method for fluid analysis. Authors validated these models qualitatively and quantitatively with VFSS images (Kikuchi et al. 2017).

The authors observed that laryngeal penetration is highly related to the tilt angle of epiglottis. These authors also concluded, and emphasised, the need to include in future studies a detailed analysis of the individual forces responsible for the epiglottic kinematics. They also observed that postural strategies can prevent aspiration by diverting the swallowed particles from the midsagittal plane at the laryngeal entry level (Kikuchi et al. 2017).

Michiwaki et al. (2019) obtained the first realistic model of organs, based on CT scan and VFSS images synchronization. They included anatomical boundaries of soft-tissue organs (tongue, soft palate, pharynx, epiglottis, larynx, oesophagus and trachea) and they used a mesh-free moving particle method to simulate bolus flow (Michiwaki et al. 2019).

This simulation showed that pathological model had a more pronounced upward movement of hyoid bone and larynx. Tongue base had more movement and occurred earlier whereas the closure of the glottis happened later. The pathological model also presented aspiration, a more spread bolus with a faster flow rate and higher quantity of pharyngeal residues (Michiwaki et al. 2019).

\section{Discussion and Conclusions}

Dysphagia can have serious clinical consequences including death. These consequences can be avoided with a correct diagnosis, a detailed evaluation and a targeted treatment. The most used instrumental methods (VFSS and FEES) include aspiration risk and inherent disadvantages of each technique (e.g.: excessive radiation or discomfort).

The swallowing computer simulation advantages have motivated several authors, over time, to develop increasingly complex and meaningful models.

Some authors investigated the importance of some anatomical and functional details for the efficacy of the swallowing process, namely epiglottis retroflexion movement and larynx elevation. Other authors have more specifically addressed the tongue biomechanics and the oral fluids containment.

Recently, authors have included more complex rheological characteristics in their studies. They have analysed the liquids viscosity and density and included Newtonian and nonNewtonian fluids (representative of thickened liquids, usually used with dysphagic patients).

Some aspects relative to the bolus flow have also been added: the gravitational force, peritalsis-like movement of the pharynx, deformability, flow resistance and frictional resistance. More recent studies have already considered the role of saliva in their models.

Regarding the characteristics included in simulations, some improvements have been proposed to previous models, namely the replacement of solid elements by shell elements, the composition of the tongue (in two parts) or the inclusion of a soft palate.

More recent papers have used data from medical images (VFSS, CT scan, MRI, and anatomical dissection images) in order to obtain realistic movement boundaries of oropharyngeal structures. 
Some authors have already included in their analysis some pathological changes of the swallowing process. The corresponding conclusions have already allowed to better understand the biomechanics underlying some clinical situations.

Future studies should continue to be based on medical imaging exams, in order to obtain meaningful results. In addition to the anatomical/structural information taken from these exams, the most frequently observed patterns of pathological changes may also be collected. Thus, the simulation of realistic pathological models may help to identify patients at higher aspiration risk, determine the most appropriate ingested viscosities for each type of pattern, and, ultimately, improve the treatment of these patients.

Regarding the simulated fluids viscosities, it will be useful to choose the most used in clinical practice, so as to be able to match the obtained conclusions with clinical practice.

Finally, in relation to the pathological model, it may be important to choose a dysfunctional pattern (rather than degenerative, e.g. age), as well as a prevalent pathology with a high dysphagia incidence (e.g. stroke).

To reduce dysphagia impact on health it is necessary a more detailed knowledge of its biomechanics and a more rigorous assessment. In turn, this assessment also needs to be safer (with less risk to the patient). Computer simulation seems to be promising to reduce clinical disadvantages and even institutional costs associated with complementary diagnostic tests.

\section{References}

Adnerhill, I., O. Ekberg, and M. E. Groher. 1989. "Determining normal bolus size for thin liquids". Dysphagia 4, no. 1 (march): 1-3. https://doi.org/10.1007/BF02407395.

Belldev Medical. n.d. "Swallowtail. Quantitative analysis of the videofluoroscopic swallow study". Accessed April 14, 2019. http://www.belldevmedical.com/swallowtail.

Chang, M. W., B. Rosendall, and B. A. Finlayson. 1998. "Mathematical modeling of normal pharyngeal bolus transport: A preliminary study". Journal of Rehabilitation Research and $\begin{array}{lllll}\text { Development } & 35, & \text { no. } & 3 & \text { (july): }\end{array}$ https://www.rehab.research.va.gov/jour/98/35/3/chang.htm.

Chang, M. W., E. Lin, and J. N. Hwang. 1999. "Contour tracking using a knowledge-based Snake algorithm to construct three-dimensional pharyngeal bolus movement". Dysphagia 14, no. 4 (august): 219-27. https://doi.org/10.1007/PL00009609.

Cook, I. J., W. J. Dodds, R. O. Dantas, B. Massey, M. K. Kern, I. M. Lang, J. G. Brausseur, and W. J. Hogan. 1989. "Opening mechanisms of the human upper esophageal sphincter". American Journal of Physiology - Gastrointestinal and Liver Physiology 257, no. 5 (november): G748-G759. https://doi.org/10.1152/ajpgi.1989.257.5.G748.

Dantas, R. O., W. J. Dodds, B. T. Massey, and M. K. Kern. 1989. "The effect of high- vs lowdensity barium preparations on the quantitative features of swallowing". American Journal of Roentgenology 153, no. 6 (december): 1191-95. https://doi.org/10.2214/ajr.153.6.1191.

de Loubens, C., A. Magnin, E. Verin, M. Doyennette, I. C. Tréléa, and I. Souchon. 2010. "A lubrication analysis of pharyngeal peristalsis: Application to flavour release". Journal of Theoretical Biology 267, no. 3 (december): 300-11. https://doi.org/10.1016/j.jtbi.2010.09.003.

de Loubens, C., A. Magnin, M. Doyennette, I. C. Tréléa, and I. Souchon. 2011. "A biomechanical model of swallowing for understanding the influence of saliva and food bolus viscosity on flavor release". Journal of Theoretical Biology 280, no. 1 (july): 180-88. https://doi.org/10.1016/j.jtbi.2011.04.016. 
Ekberg, O., and S. V. Sigurjónsson. 1982. "Movement of the epiglottis during deglutition - A cineradiographic study". Gastrointestinal Radiology 7, no. 1 (december): 101-07. https://doi.org/10.1007/BF01887619.

Fairfield, C., J. Bloomfield, P. Gillard, C. Miron, S. Naire, P. Nielsen, S. Payvandi et al. 2010. Mathematical modelling of the normal swallow. UK Mathematics in Medicine Study Groups. University of Strathclyde. https://pdfs.semanticscholar.org/efbc/2dffb02131a9164c2728661c22ac4902884e.pdf.

Farazi, M. R., B. Martin-Harris, N. M. Harandi, S. Fels, and R. Abugharbieh. 2015. "A 3D dynamic biomechanical swallowing model for training and diagnosis of dysphagia". In Proceedings International Symposium on Biomedical Imaging - 12th IEEE International Symposium on Biomedical Imaging, 1385-88. https://doi.org/10.1109/ISBI.2015.7164134.

Fink, B. R., R. W. Martin, and C. A. Rohrmann. 1979. "Biomechanics of the human epiglottis". Acta Oto-Laryngologica 87, no. 3-6: 554-59. https://doi.org/10.3109/00016487909126464.

Garon, B. R., Z. Huang, S. Hommeyer, D. Eckmann, G. A. Stern, and C. Ormiston. 2002. "Epiglottic dysfunction: Abnormal epiglottic movement patterns". Dysphagia 17, no. 1 (january): 57-68. https://doi.org/10.1007/s00455-001-0102-8.

Hayoun, P., J. Engmann, S. Mowlavi, B. Le Reverend, A. Burbidge, and M. Ramaioli. 2015. "A model experiment to understand the oral phase of swallowing of Newtonian liquids". Journal of Biomechanics 48, no. 14 (november): 3922-28. https://doi.org/10.1016/j.jbiomech.2015.09.022.

Ho, A. K., L. Tsou, S. Green, and S. Fels. 2014. "A 3D swallowing simulation using smoothed particle hydrodynamics". Computer Methods in Biomechanics and Biomedical Engineering: Imaging and Visualization 2, no. 4 (december): 237-44. https://doi.org/10.1080/21681163.2013.862862.

Ho, A. K., Y. Inamoto, E. Saitoh, S. Green, and S. Fels. 2018. "Extracting moving boundaries from dynamic, multislice CT images for fluid simulation". Computer Methods in Biomechanics and Biomedical Engineering: Imaging and Visualization 6, no. 5 (september): 539-44. https://doi.org/10.1080/21681163.2016.1188028.

Hopkin, G. B. 1967. "Neonatal and adult tongue dimensions". Angle Orthodontist 37, no. 2 (april): 132-33. https://www.angle.org/doi/abs/10.1043/00033219\%281967\%29037\%3C0132\%3ANAATD\%3E2.0.CO\%3B2.

Kahrilas, P. J., S. Lin, J. A. Logemann, G. A. Ergun, and F. Facchini. 1993. "Deglutitive tongue action: Volume accommodation and bolus propulsion". Gastroenterology 104, no. 1 (january): 152-62. https://doi.org/10.1016/0016-5085(93)90847-6.

Kahrilas, P. J., S. Lin, J. Chen, and J. A. Logemann. 1996. "Oropharyngeal accommodation to swallow volume". Gastroenterology 111, no. 2 (august): 297-306. https://doi.org/10.1053/gast.1996.v111.pm8690194.

Kikuchi, T., Y. Michiwaki, T. Kamiya, Y. Toyama, T. Tamai, and S. Koshizuka. 2015. "Human swallowing simulation based on videofluorography images using Hamiltonian MPS method". Computational Particle Mechanics 2, no. 3 (september): 247-60. https://doi.org/10.1007/s40571-015-0049-4.

Kikuchi, T., Y. Michiwaki, S. Koshizuka, T. Kamiya, and Y. Toyama. 2017. "Numerical simulation of interaction between organs and food bolus during swallowing and aspiration". Computers in Biology and Medicine 80 (january): 114-23. https://doi.org/10.1016/j.compbiomed.2016.11.017. 
Langmore, S. E. 2017. "History of fiberoptic endoscopic evaluation of swallowing for evaluation and management of pharyngeal dysphagia: Changes over the years". Dysphagia 32, no. 1 (february): 27-38. https://doi.org/10.1007/s00455-016-9775-x.

Leite, K. K. A., L. D. Mangilli, F. C. Sassi, S. C. O. Limongi, and C. R. F. de Andrade. 2014. "Ultrasonography and swallowing: a critical review of the literature". Audiology Communication Research 19 (october-december): 412-20. http://doi.org/10.1590/S231764312014000300001378.

Mackley, M. R., C. Tock, R. Anthony, S. A. Butler, G. Chapman, and D. C. Vadillo. 2013. "The rheology and processing behavior of starch and gum-based dysphagia thickeners". Journal of Rheology 57, no. 6 (november): 1533-53. https://doi.org/10.1122/1.4820494.

Manabe, N., K. Haruma, R. Nakato, H. Kusunoki, T. Kamada, and J. Hata. 2018. "New ultrasonographic screening method for oropharyngeal dysphagia: Tissue Doppler imaging". American Journal of Physiology - Gastrointestinal and Liver Physiology 314, no. 1 (january): G32-G38. https://doi.org/10.1152/ajpgi.00019.2017.

Matsuo, K., and J. B. Palmer. 2008. "Anatomy and physiology of feeding and swallowing: Normal and abnormal". Physical Medicine and Rehabilitation Clinics of North America 19, no. 4 (november): 691-707. https://doi.org/10.1016/j.pmr.2008.06.001.

Meng, Y., M. A. Rao, and A. K. Datta. 2005. "Computer simulation of the pharyngeal bolus transport of Newtonian and non-Newtonian fluids". Food and Bioproducts Processing 83, no. 4 C (december): 297-305. https://doi.org/10.1205/fbp.04209.

Michiwaki, Y., T. Kamiya, T. Kikuchi, Y. Toyama, K. Hanyuu, M. Takai, and S. Koshizuka. 2019. "Modelling of swallowing organs and its validation using Swallow Vision ${ }^{\circledR}$, a numerical swallowing simulator". Computer Methods in Biomechanics and Biomedical Engineering: Imaging and Visualization 7, no. 4 (july): 374-81. https://doi.org/10.1080/21681163.2018.1466198.

Mizunuma, H., M. Sonomura, K. Shimokasa, H. Ogoshi, S. Nakamura, and N. Tayama. 2009. "Numerical modeling and simulation on the swallowing of jelly". Journal of Texture Studies 40, no. 4 (august): 406-26. https://doi.org/10.1111/j.1745-4603.2009.00189.x.

Mowlavi, S., J. Engmann, A. Burbidge, R. Lloyd, P. Hayoun, B. Le Reverend, and M. Ramaioli. 2016. "In vivo observations and in vitro experiments on the oral phase of swallowing of Newtonian and shear-thinning liquids". Journal of Biomechanics 49, no. 16 (december): 3788-95. https://doi.org/10.1016/j.jbiomech.2016.10.011.

Napadow, V. J., R. D. Kamm, and R. J. Gilbert. 2002. "A biomechanical model of sagittal tongue bending". Journal of Biomechanical Engineering 124, no. 5 (october): 547-56. https://doi.org/10.1115/1.1503794.

Newman, R., N. Vilardell, P. Clavé, and R. Speyer. 2016. "Effect of bolus viscosity on the safety and efficacy of swallowing and the kinematics of the swallow response in patients with oropharyngeal dysphagia: White paper by the European Society for Swallowing Disorders (ESSD)". Dysphagia 31, no. 2 (april): 232-49. https://doi.org/10.1007/s00455-016-9696-8.

Nicosia, M. A., and J. Robbins. 2001. "The fluid mechanics of bolus ejection from the oral cavity". Journal of Biomechanics 34, no. 12 (december): 1537-44. https://doi.org/10.1016/S0021-9290(01)00147-6.

Nicosia, M. A. 2007. "A planar finite element model of bolus containment in the oral cavity". Computers in Biology and Medicine 37, no. 10 (october): 1472-78. https://doi.org/10.1016/j.compbiomed.2007.01.007. 
Nicosia, M. A. 2013. "Theoretical estimation of shear rate during the oral phase of swallowing: Effect of partial slip". Journal of Texture Studies 44, no. 2 (april): 132-39. https://doi.org/10.1111/jtxs.12005.

Ono, T., K. Hori, K. i Tamine, and Y. Maeda. 2009. "Evaluation of tongue motor biomechanics during swallowing-From oral feeding models to quantitative sensing methods". Japanese Dental Science Review 45, no. 2 (september): 65-74. https://doi.org/10.1016/j.jdsr.2009.03.005.

Pearson, W. G., Jr., B. K. Taylor, J. Blair, and B. Martin-Harris. 2016. "Computational analysis of swallowing mechanics underlying impaired epiglottic inversion". Laryngoscope 126, no. 8 (august): 1854-58. https://doi.org/10.1002/lary.25788.

Perlman, A. L., B. M. Booth, and J. P. Grayhack. 1994. "Videofluoroscopic predictors of aspiration in patients with oropharyngeal dysphagia". Dysphagia 9, no. 2 (march): 90-95. https://doi.org/10.1007/BF00714593.

Poorjavad, M., S. Talebian, N. Nakhostin Ansari, and Z. Soleymani. 2017. "Surface electromyographic assessment of swallowing function". Iranian Journal of Medical Sciences 42, no. 2 (march): 194-200. https://www.ncbi.nlm.nih.gov/pmc/articles/PMC5366368/.

Santamato, A., F. Panza, V. Solfrizzi, A. Russo, V. Frisardi, M. Megna, M. Ranieri, and P. Fiore. 2009. "Acoustic analysis of swallowing sounds: A new technique for assessing dysphagia". Journal of Rehabilitation Medicine 41, no. 8 (july): 639-45. https://doi.org/10.2340/16501977-0384.

Seo, H. G., B. M. Oh, J. H. Leigh, and T. R. Han. 2014. "Correlation varies with different time lags between the motions of the hyoid bone, epiglottis, and larynx during swallowing". Dysphagia 29, no. 5 (september): 591-602. https://doi.org/10.1007/s00455-014-9550-9.

Seo, H. G., B. M. Oh, and T. R. Han. 2016. "Swallowing kinematics and factors associated with laryngeal penetration and aspiration in stroke survivors with dysphagia". Dysphagia 31, no. 2 (april): 160-68. https://doi.org/10.1007/s00455-015-9670-x.

Sonomura, M., H. Mizunuma, T. Numamori, H. Michiwaki, and K. Nishinari. 2011. "Numerical simulation of the swallowing of liquid bolus". Journal of Texture Studies 42, no. 3 (june): 203-11. https://doi.org/10.1111/j.1745-4603.2011.00287.x.

Spadotto, A. A., A. R. Gatto, P. C. Cola, A. N. Montagnoli, A. O. Schelp, R. G. Da Silva, S. Yamashita, J. C. Pereira, and M. A. C. De Arruda Henry. 2008. "Swallowing quantitative analysis software". Radiologia Brasileira 41, no. 1 (january): 25-28. https://doi.org/10.1590/S0100-39842008000100008.

Tasko, S. M., R. D. Kent, and J. R. Westbury. 2002. "Variability in tongue movement kinematics during normal liquid swallowing". Dysphagia 17, no. 2 (march): 126-38. https://doi.org/10.1007/s00455-001-0112-6.

Vandaele, D. J., A. L. Perlman, and M. D. Cassell. 1995. "Intrinsic fibre architecture and attachments of the human epiglottis and their contributions to the mechanism of deglutition". Journal of Anatomy 186, no. 1 (february): 1-15. https://www.ncbi.nlm.nih.gov/pmc/articles/PMC1167268/.

Vilardell, N., L. Rofes, V. Arreola, A. Martin, D. Muriana, E. Palomeras, O. Ortega, and P. Clavé. 2017. "Videofluoroscopic assessment of the pathophysiology of chronic poststroke oropharyngeal dysphagia". Neurogastroenterology and Motility 29, no. 10 (october): 1-8. https://doi.org/10.1111/nmo.13111. 